\title{
Factores asociados a la automedicación frente a COVID-19 en usuarios de las redes sociales - PERÚ.
}

Factors associated with self-medication against COVID-19 in users of social networks - Peru.

Vilma Amparo Junchaya Yllescas 1,a, Diana Esmeralda Andamayo Flores 1,b, Karen Janet Ayala Guevara ${ }^{1, c}$, Aracely Janett Maravi Cabrera ${ }^{1, d}$, Pedro Yvan Saenz Rivera ${ }^{2, e}$

\section{RESUMEN}

Objetivo: Identificar los factores asociados a la automedicación con fármacos relacionados a COVID-19 en pobladores que hacen uso de las redes sociales en el Perú. Material y Métodos: Se realizó un estudio de corte transversal, en una muestra intencionada de 1068 usuarios del Facebook en el Perú, aplicándose para la obtención de datos, la técnica de encuesta y el instrumento un cuestionario digital de Google que considera a la dimensión económica y cultural. Resultados: Se halló como características sociodemográficos predominantes, el sexo femenino 82,4\%, de 18 a 22 años en el 28,3\%, de educación universitaria en el 57,8 \%; también como hallazgos de factores económicos, un $34,8 \%$ son de ocupación profesional, de ingreso económico regular en el 39,5\%, se consideran de condición económica media en el $64,8 \%$ y como factores culturales un $84,3 \%$ se automedica, en frecuencia de rara vez en el $65 \%$ y lo hacen por recomendación del personal de salud en el 47,1\%. Conclusiones: La automedicación fue alta en los usuarios del Facebook en el Perú y que tiene relación significativa con mayor número de factores culturales como: Fuentes para automedicarse, las razones y frecuencia del consumo es lo que más predomina en los usuarios del Facebook-Perú constituyendo un grave problema de salud pública.

PALABRAS CLAVE: Automedicación, factores, COVID-19.

\section{SUMMARY}

Objective: To identify the factors associated with self-medication with drugs related to COVID-19 in the population using social networks - Peru. Material and Methods: A cross-sectional study was carried out in a purposive sample of 1068 users of Facebook in Peru, applying the survey technique to obtain data and the instrument was a Google digital questionnaire that considers the economic and cultural dimension. Results: The predominant sociodemographic characteristics were found to be female $82.4 \%, 18$ to 22 years old in $28.3 \%$, university education in $57.8 \%$; also, as findings of economic factors, $34.8 \%$ are of professional occupation, of regular economic income in $39.5 \%$, they are considered of average economic condition in $64.8 \%$ and as cultural factors $84.3 \%$ self-medicate, in frequency of rarely in $65 \%$ and they do it on the recommendation of health personnel in $47.1 \%$. Conclusion: Self-medication

\footnotetext{
${ }^{1}$ Universidad Privada de Huancayo Franklin Roosevelt. Huancayo, Perú.

${ }^{2}$ Universidad Nacional Federico Villareal. Lima, Perú.

a Docente; Químico Farmacéutico; Magister; ORCID ID: 0000-0002-5035-2157

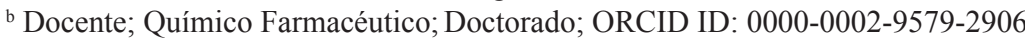

${ }^{c}$ Docente; Químico Farmacéutico; Magister; ORCID ID: 0000-0002-3812-2359

d Docente; Químico Farmacéutico; Magister; ORCID ID: 0000-0002-2068-3924

e Docente; Lic. en Estadística; Magister; ORCID ID: 0000-0002-8131-4997
} 
was high in Facebook-Peru users and it is significantly related to a greater number of cultural factors such as: sources for self-medication, the reasons and frequency of consumption is what predominates most in Facebook-Peru users, constituting a serious public health problem.

Keywords: Self-medication, factors, COVID-19

\section{INTRODUCCIÓN}

La Organización Mundial de la Salud define a la automedicación como una selección y el uso de los medicamentos por parte de la población, con el propósito de prevenir, aliviar o tratar síntomas o enfermedades leves que ellas mismas puedan identificar. Este organismo internacional estima que más de la mitad de los medicamentos a nivel mundial se prescriben, dispensan o venden de manera inapropiada, asimismo, las formas más comunes de uso inadecuado de los medicamentos incluyen la polifarmacia, la excesiva aplicación de inyecciones, la incorrecta utilización de antibióticos, la falta de prescripción de acuerdo con las guías clínicas, la auto prescripción y la falta de adherencia a los tratamientos (1).

La automedicación es un riesgo debido a múltiples factores que pueden traer consecuencias negativas a corto y largo plazo en la vida de una persona, por lo tanto, todos los medicamentos deben ser administrados en dosis y durante una cantidad de días determinados por un especialista en la salud y no por una recomendación pasajera de algún familiar, amigo, etc. Además de acuerdo a la base de datos del Centro Nacional de Farmacovigilancia y Tecno vigilancia, se han reportado más de 300 casos de sospechas de reacciones adversas a medicamentos empleados para el tratamiento de personas afectadas por el COVID-19, siendo lo más frecuente los trastornos gastrointestinales relacionados a la ingesta de Ivermectina (2).

En la actualidad no se recomienda ningún medicamento para prevenir el coronavirus y el tratamiento indicado se determinará luego de una evaluación médica y de acuerdo a los síntomas de cada paciente. Asimismo, las personas asintomáticas no necesitan tomar algún medicamento, sino cumplir con el aislamiento domiciliario y mantenerse alerta a los síntomas graves de la enfermedad como fiebre persistente, tos, insuficiencia respiratoria y acudir al médico cuando esto se presente (3).

Así también se resalta 1 investigación de Almeida et al., (4), en la cual se encontró que la automedicación fue abiertamente reconocida por el $56,60 \%$ de los encuestados. El patrón de alta escolaridad se relaciona a la práctica de automedicación en la población estudiada y se caracteriza por la compra sin receta médica en el caso de patologías leves, principalmente debido a la falta de tiempo para acudir al médico y por los bajos costos de las especialidades farmacéuticas genéricas.

Igualmente, a la fecha, no existe un tratamiento eficaz para COVID-19, que es una enfermedad pandémica, causada por un nuevo coronavirus llamado SARS-CoV-2. donde muchas personas practican la automedicación, la ausencia de una cura para el COVID-19 y la progresión constante de la enfermedad requiere una evaluación de los factores de automedicación en el contexto de la pandemia.

\section{MATERIAL Y MÉTODOS}

Para esta investigación fue utilizado el estudio de nivel descriptivo y de tipo no experimental, la población de la investigación estuvo constituida por 25 millones de usuarios del Facebook Perú, con una muestra no probabilística intencionada de corte transversal, de 1068 usuarios; para la recolección de datos fue creado un fanpage que contenía el formulario digital de Google como instrumento y técnica de encuesta auto aplicada; para mediar la confiabilidad del instrumento se procedió a cuantificar la estabilidad por test-retest, se midió de manera univariada la correlación de las respuestas mediante el coeficiente de contingencia nominal encontrándose valores entre 0,617 y 0,866 los cuales son valores adecuados según la literatura consultada (5), además también se cuantificaron los índices de Kappa por cada ítem, superándose el valor de 0,50. Los resultados fueron analizados estadísticamente, para observar la contrastación de los objetivos planteados, los mismos que se prestan en las tablas, gráficos y otros, para su respectiva interpretación metodológica y temática.

\section{RESULTADOS}

Se recibieron 1068 encuestas correctamente llenadas por los usuarios de Facebook Perú. Siendo 
las características sociodemográficas de la muestra investigada, del sexo femenino, $82,4 \%$ de edades entre 18 a 22 años, y mayor a 32 años el $27,9 \%$, de educación universitaria el 57,8\%. Asimismo, los factores económicos asociados a la automedicación son: de ocupación profesionales $34,8 \%$, que tienen sueldo regular $39,5 \%$, son de clase media $64,8 \%$, consumen medicamentos sin prescripción médica $84,3 \%$ y se automedican rara vez $65,0 \%$.

Del mismo modo, los factores culturales asociados a la automedicación son: La fuente de recomendación para automedicarse, que corresponde al personal de salud 47,1 \%, como razones de automedicación resalta, el no tener tiempo para ir al médico $37,6 \%$,

Los resultados obtenidos se muestran en las siguientes tablas:

Características Sociodemográficas: Tabla 1, tabla 2 y tabla 3

Factor económico: Tabla 4, tabla 5 y tabla 6.

Factores culturales: Tabla7, tabla 8, tabla 9 y tabla 10.

Tabla 1. Sexo

\begin{tabular}{lcc}
\hline Sexo & Frecuencia & Porcentaje \\
\hline Femenino & 880 & $82,4 \%$ \\
Masculino & 188 & $17,6 \%$ \\
Total & 1068 & $100 \%$ \\
\hline Fuente: Formulario Digital & n: 1068
\end{tabular}

Tabla 2. Edad

\begin{tabular}{lcc}
\hline Edad & Frecuencia & Porcentaje \\
\hline $18-22$ años & 302 & $28,3 \%$ \\
$23-27$ años & 238 & $22,3 \%$ \\
$28-32$ años & 230 & $21,5 \%$ \\
Mayor a 32 años & 298 & $27,9 \%$ \\
Total & 1068 & $100 \%$ \\
\hline Fuente: Formulario Digital & n: 1068 &
\end{tabular}

Tabla 3. Grado de Instrucción

\begin{tabular}{lcc}
\hline Grado de instrucción & Frecuencia & Porcentaje \\
\hline Educación Primaria & 10 & $0,9 \%$ \\
Educación Secundaria & 56 & $5,2 \%$ \\
Educación Superior Técnica & 386 & $36,1 \%$ \\
Educación universitaria & 617 & $57,8 \%$ \\
Total & 1068 & $100 \%$ \\
\hline Fuente: Formulario Digital & \multicolumn{2}{c}{ n: 1068}
\end{tabular}

Visionarios en ciencia y tecnología 2021; 6:89-94.
Tabla 4. Ocupación

\begin{tabular}{lcc}
\hline Ocupación & Frecuencia & Porcentaje \\
\hline Ama de casa & 74 & $6,9 \%$ \\
Profesional & 372 & $34,8 \%$ \\
Empleados & 184 & $17,2 \%$ \\
Desempleado & 80 & $7,5 \%$ \\
Otros & 359 & $33,6 \%$ \\
Total & 1068 & $100 \%$ \\
\hline Fuente: Formulario Digital & n: 1068
\end{tabular}

Tabla 5. Ingreso económico

\begin{tabular}{lcc}
\hline Ingreso econónico & Frecuencia & Porcentaje \\
\hline No cuenta con un & 380 & $35.6 \%$ \\
sueldo & 36 & $3.4 \%$ \\
Sueldo bueno & 422 & $39.5 \%$ \\
Sueldo regular & 230 & $21.5 \%$ \\
Sueldo bajo & 1068 & $100 \%$ \\
Total & \multicolumn{2}{c}{$\mathrm{n}: 1068$}
\end{tabular}

Tabla 6. Clase socio econónica

\begin{tabular}{lcc}
\hline $\begin{array}{l}\text { Clase socio } \\
\text { econónico }\end{array}$ & Frecuencia & Porcentaje \\
\hline Alta & 12 & $1.1 \%$ \\
Media & 692 & $64.8 \%$ \\
Baja & 364 & $34.1 \%$ \\
Total & 1068 & $100 \%$ \\
\hline Fuente: Formulario Digital & \multicolumn{2}{c}{ n: 1068}
\end{tabular}

Tabla 7. ¿Alguna vez ha consumido medicamentos sin prescripción médica?

\begin{tabular}{lcc}
\hline $\begin{array}{l}\text { Consumo de } \\
\text { medicamentos sin R P. }\end{array}$ & Frecuencia & Porcentaje \\
\hline $\mathrm{Si}$ & 900 & $84,3 \%$ \\
$\mathrm{No}$ & 168 & $15,7 \%$ \\
Total & 1068 & $100 \%$ \\
\hline Fuente: Formulario Digital & \multicolumn{2}{c}{ n: 1068}
\end{tabular}

Tabla 8. ¿Con qué frecuencia ha tomado medicamentos sin prescripción médica durante esta pandemia COVID-19?

\begin{tabular}{lcc}
\hline Frecuencia de consumo & Frecuencia & Porcentaje \\
\hline Habitualmente & 172 & $16,1 \%$ \\
Rara vez & 694 & $65,0 \%$ \\
Nunca & 202 & $18,9 \%$ \\
Total & 1068 & $100 \%$ \\
\hline Fuente: Formulario Digital & \multicolumn{2}{c}{ n: 1068}
\end{tabular}


Tabla 9. Fuentes de recomendación para automedicación COVID-19

\begin{tabular}{lcc}
\hline Recomendación & Frecuencia & Porcentaje \\
\hline Familia & 155 & $14,5 \%$ \\
Amigos & 53 & $5,0 \%$ \\
Personal de Salud & 503 & $47,1 \%$ \\
Medios de Comunicación & 96 & $9,0 \%$ \\
Personal de la Botica & 261 & $24,4 \%$ \\
Total & 1068 & $100 \%$ \\
\hline Fuente: Formulario Digital & n: 1068
\end{tabular}

que el $10 \%$ de las enfermeras se automedican por las actividades que realizan, además que existe un $59 \%$ de otros profesionales que sugieren rápidamente a la automedicación, de igual forma Pereira et al., (9) concluye que hay una alta tasa de automedicación entre los profesionales de la salud. No obstante, se contradice con el trabajo de Sadio et al., (10), quienes consideran que la automedicación a menudo se ha asociado con un nivel educativo más bajo

El hecho que los profesionales tengan acceso a los medicamentos definitivamente facilita la

Tabla 10. Razones de automedicación COVID-19

\begin{tabular}{lcc}
\hline Razón de automedicarse & Frecuencia & Porcentaje \\
\hline No tengo el dinero para acudir a un Servicio de Salud. & 227 & $21,3 \%$ \\
No tengo tiempo para ir al médico. & 402 & $37,6 \%$ \\
No cuento con un Servicio de Salud. & 182 & $17,0 \%$ \\
No creo que sea necesario consultar al médico. & 164 & $15,4 \%$ \\
Tiene desconfianza en los médicos. & 92 & $8,6 \%$ \\
Total & 1068 & $100 \%$ \\
\hline Fuente: Formulario Digital & n: 1068 &
\end{tabular}

\section{DISCUSIÓN}

Dentro de las características sociodemográficas asociados a la automedicación, de los 1068 usuarios que respondieron el formulario digital, un $82,4 \%$ son de sexo femenino, 18 a 22 años en el 28,3\% y de educación universitaria en el 57,8 \%. Definitivamente ante el miedo y la ansiedad crecientes generados por la pandemia de la COVID-19 y por demora en la aparición de una vacuna eficaz y justamente a los tiempos de investigación para el desarrollo de una vacuna, es cuando muchas personas optaron casi de manera instintiva, por automedicarse con diferentes sustancias. En ese contexto, tradicionalmente la automedicación se ha extendido durante la pandemia, siendo una práctica riesgosa e irresponsable, pudiendo ocasionar desde una resistencia bacteriana hasta una intoxicación por medicamentos, por ello la existencia de un sistema de salud colapsado en nuestro país (6).

También en este trabajo se encontró que un 84,3 $\%$ se automedican y se asemeja al estudio de Alayo el al., (7), quienes hallaron que el $46,3 \%$ de pobladores se automedican. Al analizar los factores económicos se demuestra que pertenecen a la ocupación profesionales quienes se automedican en $34 ., 8 \%$, lo cual coincide con el estudio de Abad (8), quien señala automedicación, sumado a ello su jornada de trabajo los condiciona para esa práctica equivocada, ya que la complexidad del trabajo, muchas veces el profesional tiene más de un empleo, pasan por situaciones estresantes y consecuentemente desarrollan alguna sintomatología, haciendo la automedicación un medio de facilitar su vida.

Con respecto al ingreso económico el $39.5 \%$ cuenta con un sueldo regular, el cual se asemeja con Bernal et al., (11), quienes hallaron como resultado que de los comerciantes el $37 \%$ cuentan con ingreso mensual inferior a 1000 soles; en relación a la clase socioeconómica se encontró como resultado que el $64,8 \%$ pertenece a la clase media, lo que difiere con la investigación de Cerna et al., (12), quienes concluyen que el nivel socioeconómico cultural de los habitantes del asentamiento humano Cerrito la Libertad es relativamente bajo y existe cierto grado de asociación entre la automedicación y el nivel socioeconómico. Sin embargo, con estos resultados queda demostrado que nivel económico no es determinante para la automedicación.

Del mismo modo, estos resultados señalan que una de las razones que podría explicar el automedicarse es la clase socioeconómica y el ingreso económico, ya 
que no todas las personas poseen la misma capacidad de pago ante los servicios sanitarios y muchos de ellos se ven en la necesidad de automedicarse, lo que trae como consecuencia deterioro de la salud y serio problema de salud pública.

Seguidamente en cuanto a los factores culturales, los usuarios responden que se automedicaron, como fuente de información por recomendación del personal del salud $47,1 \%$, este resultado se corrobora con la investigación de Ninayahuar et al., (13) quienes demuestra que la recomendación para el autoconsumo fue por un profesional de salud no médico en el $11,4 \%$, asimismo se reafirma con el estudio de Puma (14), quien halló que los usuarios de un mercado se automedicaban por recomendación del personal farmacéutico en un $63,4 \%$, de la misma forma se asemeja con la investigación de Matos (15), quien demostró que los usuarios de un establecimiento farmacéutico se automedicaban por recomendación del personal de salud $60,76 \%$.

También, concerniente a las razones y motivos de la automedicación, manifiestan que lo hacen por no tener tiempo para ir al médico $37,6 \%$, lo cual coincide con el estudio de Cuya et al., (16), quien menciona que la causa principal para no acudir al médico fue la falta de tiempo en $81 \%$, asimismo, se corrobora con el estudio de Alba et al., (17), muestran que los estudiantes de un área de salud se automedicaban por la falta de tiempo $42,1 \%$, de la misma forma, en el estudio de Bernal et al., (11), corrobora que de los adultos encuestados refieren que se automedican porque no dispone de tiempo para ir al médico.

Estos resultados indican, que necesariamente se necesitaba un tiempo de atención para una consulta médica; sin embargo, la coyuntura actual vivida no permitía ir con esa facilidad y más aún si los servicios hospitalarios se encontraban saturados con enfermos de coronavirus, debido a ello que los usuarios buscaron una atención rápida, siendo lo más rápido asistir a una botica o farmacia para automedicarse.

\section{CONCLUSIONES}

En conclusión, nuestros resultados revelaron que los factores asociados a la automedicación con fármacos relacionados a COVID-19 de un total de 1068 pobladores, se halló como características sociodemográficos predominantes, del sexo femenino $82,4 \%$, de 18 a 22 años en el $28,3 \%$, de educación universitaria en el 57,8\%; también como hallazgos de factores económicos, un 34,8 \% fueron de ocupación profesional, de ingreso económico regular en el 39,5 $\%$, se consideró de condición económica media en el $64,8 \%$ y como factores culturales un $84,3 \%$ se automedica, en frecuencia de rara vez en el $65 \%$ y lo hacen por recomendación del personal de salud en el $47,1 \%$. Por lo que, la automedicación fue alta en los usuarios del Facebook -Perú y hubo relación significativa con mayor número de factores culturales como las fuentes para automedicarse, las razones y frecuencia del consumo es lo que más predomina en los usuarios constituyendo un grave problema de salud pública.

\section{REFERENCIAS}

1. Administración Nacional de Medicamentos, Alimentos y Tecnología Médica. Cuidá tu salud usá responsablemente los medicamentos. Buenos Aires: Administración Nacional de Medicamentos, Alimentos y Tecnología Médica;2019. (Citado el 31 de Marzo del 2021). Disponible en: http://www. anmat.gov.ar/comunicados/Usa_responsablemente los_medicamentos.pdf

2. Instituto Carrión. Los peligros de la automedicación. Lima: Instituto Carrión; 2021. (Citado el 31 de Marzo del 2021). Disponible en: Disponible en la URL: https://www.acarrion.edu.pe/noticia_detalle/covid19-peligros-de-la-automedicacion/.

3. Ministerio de Salud. Automedicación puede agravar salud de pacientes con Covid-19. Lima: Ministerio de Salud ; 2021. (Citado el 31 de Marzo del 2021). Disponible en: https://www.gob.pe/institucion/ minsa/noticias/297083-automedicacion-puedeagravar-salud-de-pacientes-con-covid-19

4. Almeida M, Priego H. Automedicación de medicamentos genéricos en usuarios de farmacias en un municipio mexicano. Revista de la Facultad de Ciencias de la Salud de la Universidad del Cauca. 2020; 22 (1): $24-32$.

5. Hernández R, Mendoza C. Metodología de la investigación las rutas cuantitativa, cualitativa y mixta. Ciudad de Mexico: Mc Graw Hill;2018.

6. Mera A. Delgado-Noguera M. Conocimientos y necesidades del personal de salud sobre elementos de protección personal durante la pandemia por COVID-19 en el Cauca. Revista de la Facultad de Ciencias de la Salud Universidad del Cauca. 2020; 22 (1): $16-23$.

7. Alayo A, Contreras M. (2021). Influencia social y automedicación en los pobladores de Huamachuco. Tesis para Título Profesional en enfermería. Trujillo, Perú: Universidad Nacional de Trujillo; 2021. (Citado el 31 de julio del 2021). Disponible en: https://dspace. un itru.edu.pe/bitstrea m/handle/ 
UNITRU $/ 18244 / 1988$.pdf? sequence $=1 \&$ is Allowed $=\mathrm{y}$

8. Abad M.. Determinantes asociados a la automedicación en profesionales de enfermería del hospital de Tingo María.Tesis de Licenciatura. Huanuco, Perú: Universidad de Huánuco; 2021. (Citado el 31 de Marzo del 2021). Disponible en: http://distancia.udh.edu.pe/handle/123456789/2895.

9. Pereira W, Gonçalves P, Iara de Jesús C, et al Automedicação entre profissionais de saúde do município de Fátima/BA. Automedicação entre profissionais de saúde do município de Fátima/BA. Revista Eletrônica Acervo Saúde. 2029; 11 (12). DOI: $10.25248 /$ reas.e534.2019

10. Sadio A, Gbeasor F, Konu R, et al. Evaluación de las prácticas de automedicación en el contexto del brote de COVID-19 en Togo. BMC Public Health. 2021;21(1):58. doi: 10.1186/s12889-020-10145-1

11. Bernal C, Gallardo O.. Diferencias en automedicación durante la pandemia covid-19 entre los comerciantes del mercado central de Huacho y mercado municipal de Huaura Febrero - Abril 2021. Tesis de Grado. Lima, Perú: Universidad Maria Auxiliadora; 2021.

12. Cerna D, Salvatierra A. Automedicación y nivel socioeconómico cultural en el manejo de la covid-19 por pobladores del asentamiento humano cerrito la Libertad. Tesis de Grado. Lima, Perú: Universidad María Auxiliadora; 2021.

13. Ninayahuar N, Ramos A. Determinar el grado de conocimiento de automedicación en pacientes de la tercera edad con enfermedades crónicas en tiempos de COVID-19 en Cadenas de Farmacia - Lima 2020. Tesis de Grado. Huancayo, Perú: Universidad Roosevelt;2021. (Citado el 31 de Marzo del 2021). Disponible en: https://repositorio.uroosevelt.edu.pe/ handle/ROOSEVELT/362
14. Puma S, Rivera S. La automedicación en la pandemia por COVID-19 en pobladores de dos mercados más importantes de SJL - Lima 2020. Tesis de Grado. Huancayo, Perú: Universidad Roosevelt;2020. (Citado el 31 de Marzo del 2021). Disponible en: https://repositorio.uroosevelt.edu.pe/handle/ ROOSEVELT/246

15. Matos J, Pariona E. Evaluación de la Automedicación con Antibióticos en COVID-19 en adultos usuarios de Facebook Perú. Tesis de Grado. Huancayo, Perú: Universidad Roosevelt; 2020. ( (Citado el 31 de Marzo del 2021). Disponible en: https://repositorio. uroosevelt.edu.pe/handle/ROOSEVELT/362

16. Cuya A, Macotela P. Automedicación en trabajadores del mercado santa rosa chorrillos en los tiempos de COVID-19 entre los meses de Agosto - Octubre 2020. Tesis de Grado. Huancayo, Perú: Universidad Roosevelt;2021. (Citado el 31 de Marzo del 2021). Disponible en: https://renati.sunedu.gob.pe/handle/ sunedu/3016278.

17. Alba L, Papaqui A, Castillo N, Medina T, Papaqui H, Sánchez A. Principales causas de automedicación en estudiantes del área de la salud. Revista CONAMED. 2020; 25(1): 3-9. (Citado el 31 de Marzo del 2021). Disponible en: https://www.medigraphic. $\mathrm{com} / \mathrm{cgi}$-bin/new/resumen.cgi?IDARTICULO= 92889

Recibido: 22/08/2021

Aceptado: 19/11/2021 